\title{
De novo generation of Lettuce infectious yellows virus defective RNAs in protoplasts
}

\author{
LUIS RUBIO, TONGYAN TIAN, HSIN-HUNG YEH, YANNIS LIVIERATOS AND BRYCE W. FALK* \\ Department of Plant Pathology, 1 Shields Ave., University of California, Davis, CA 95616, USA
}

\section{SUM MARY}

Lettuce infectious yellows virus (LIYV)-infected plants contain a heterogeneous population of defective RNAs (D RNAs) derived from LIYV genomic RNA 2. To partly address how LIYV D RNAs are generated, in vitro synthesized transcripts corresponding to the LIYV genomic RNAs 1 and 2 were inoculated to protoplasts, and these were analysed for genomic and D RNAs. De novo generated D RNAs were readily detected by $48 \mathrm{~h}$ post-inoculation. Furthermore, when separate aliquots from the same protoplast preparation were separately inoculated with aliquots of the same LIYV RNA 1 and RNA 2 transcript preparations, different D RNA populations were detected in each. Thus, different D RNAs arose de novo within separate protoplast samples. Nucleotide sequence analysis of some de novo LIYV D RNAs revealed that they have a similar structure to the LIYV D RNAs described previously from whole plants, and to those of other plant viruses, consisting of one large internal deletion of the LIYV genomic RNA 2, but retaining $5^{\prime}$ and $3^{\prime}$ terminal sequences. However, one of the LIYV D RNAs had two non-contiguous internal deletions.

\section{INTRODUCTION}

Defective RNAs (D RNAs) are deletion forms of virus genomic RNAs that retain the replication signals but require the parental virus for replication. D RNAs have been reported for animal viruses (Holland, 1990) and for several plant viruses in the following genera: Carmovirus, Tombusvirus, Potexvirus, Hordeivirus, Rhabdovirus, Furovirus, Bromovirus, Cucumovirus, Tobravirus, Pomovirus, and Closterovirus (Ayllón et al., 1999; Damayanti et al., 1999; Graves et al., 1996; Torrance et al., 1999; White and Morris, 1999). Recently, we also found D RNAs associated with genomic RNA 2 of Lettuce infectious yellows virus (LIYV), the type member of the genus Crinivirus (Rubio et al., 2000).

The LIYV genome consists of two large, positive-sense, singlestranded RNAs. RNA 1 encodes for proteins required for replica-

*Correspondence: E-mail: bwfalk@ucdavis.edu tion and RNA 2 contains the closterovirus hallmark gene array and encodes for proteins including the two capsid proteins, the HSP70 homologue protein (HSP70h), and other proteins with functions not yet known (Klaassen et al., 1995; Yeh et al., 2000). Each of the LIYV D RNAs previously analysed by us retained both the $3^{\prime}$ and $5^{\prime}$ termini of LIYV RNA 2, but had one extensive internal deletion. Within LIYV-infected plants, these D RNAs exist as a heterogeneous population, differing in size and deletion location (Rubio et al., 2000). Our analyses suggested that some of these D RNAs could be encapsidated and transmitted to other plants (along with virions containing RNAs 1 and 2) by the whitefly Bemisia tabaci Genn. In vitro synthesized transcripts corresponding to two of these LIYV RNA 2 D RNAs replicated when coinoculated to protoplasts with LIYV RNAs 1 and 2, or with only LIYV RNA 1, and did not noticeably interfere with LIYV genomic RNA 1 and RNA 2 replication (Rubio et al., 2000).

In this paper, we address in part the origin of LIYV RNA 2 D RNAs. For this purpose, infectious transcripts were synthesized in vitro from cDNA clones of full-length genomic LIYV RNA 1 and RNA 2 and inoculated to protoplasts. De novo D RNAs were detected at $48 \mathrm{~h}$ post-inoculation (p.i.). We analysed the D RNA populations generated in different protoplast preparations inoculated with specific LIYV transcripts.

\section{RESULTS}

\section{Detection of LIYV D RNAs generated de novo}

Northern blot analysis of RNAs from LIYV RNA 1 and 2 transcriptinoculated protoplasts showed LIYV RNA 2 genomic and subgenomic RNAs (Fig. 1). However, LIYV RNA 2 species which did not correspond in size with the genomic or subgenomic RNAs were also observed. To better understand the nature of these RNAs, we analysed three protoplast replicates inoculated independently with equivalent amounts of LIYV RNA 1 and RNA 2 transcripts. Because these were separately inoculated, we considered each as a distinct LIYV protoplast RNA replicate. When RNAs from these protoplasts were analysed by Northern hybridization using a probe corresponding to LIYV RNA $23^{\prime}$ terminal sequence, we found one dominant LIYV RNA species corresponding to LIYV 


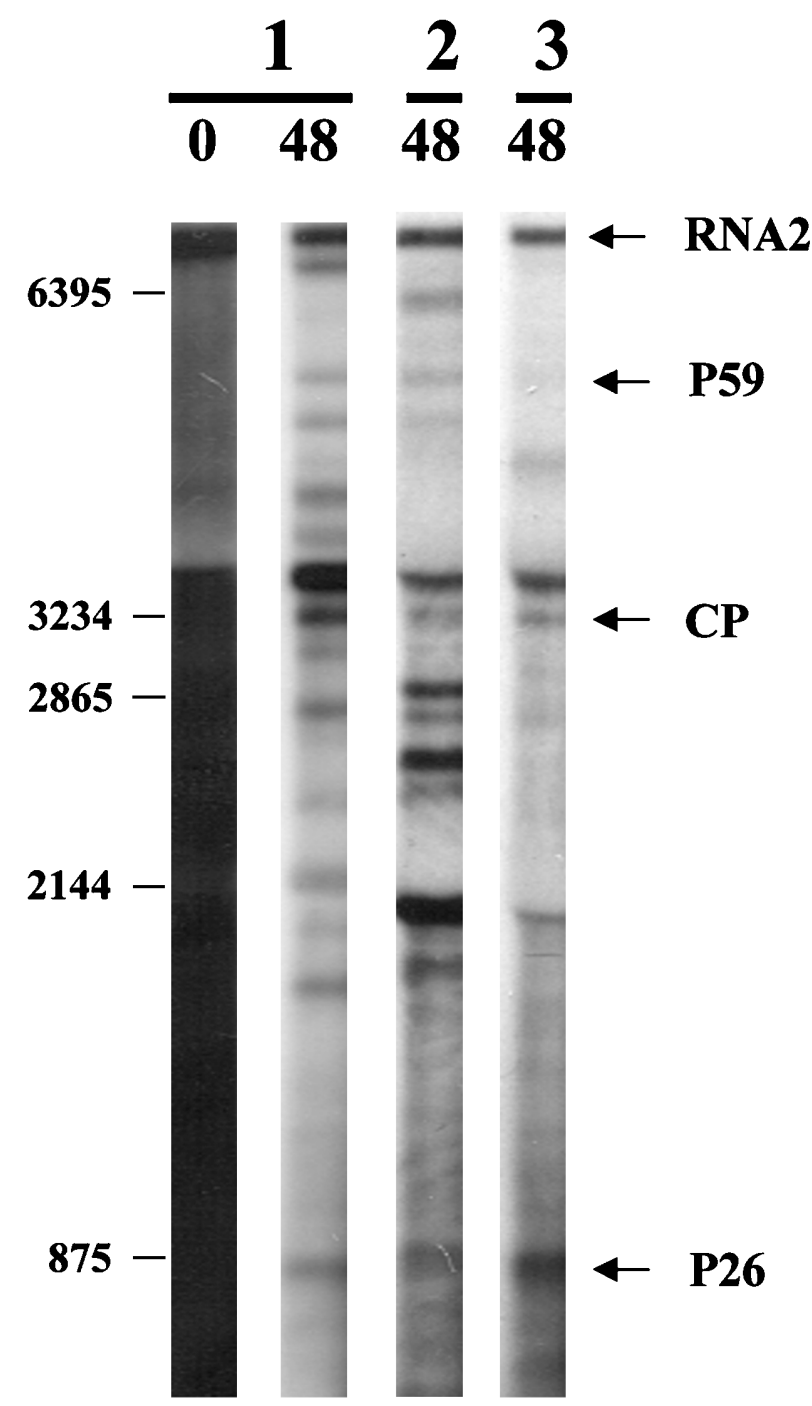

Fig. 1 Northern blot analysis of total RNAs extracted from Nicotiana tabacum protoplasts inoculated with transcripts obtained from CDNA clones of Lettuce infectious yellows virus (LIYV) genomic RNA 1 and RNA 2. Three independent inoculations were analysed and are indicated as 1, 2, and 3. Total RNAs were extracted from protoplasts at $0 \mathrm{~h}$ (inoculation 1 , lane 0 ) and at $48 \mathrm{~h}$ (inoculations 1, 2, and 3, lanes 48) post-inoculation. Hybridizations were performed using a DIG-labelled RNA probe corresponding to LIYV RNA 2 3' terminus (see probe position in Fig. 2). Positions of marker RNAs are given on the left; the numbers represent the sizes in nucleotides. Arrows on the right indicate the positions of LIYV genomic RNA 2 and subgenomic RNAs corresponding to the indicated open reading frames (ORFs) (see Fig. 2).

genomic RNA 2, as well as an intense smear representing incomplete or degraded transcripts at time 0 (at 0 h p.i.; Fig. 1, inoculation 1, lane 0). Samples analysed at $24 \mathrm{~h}$ p.i. showed almost no detectable LIYV genomic RNA 2 (not shown, but see Yeh et al., 2000). However, analysis of RNAs extracted at $48 \mathrm{~h}$ p.i. showed distinct LIYV RNAs corresponding to RNA 2 genomic and subgenomic RNAs, plus a number of RNAs which did not correspond

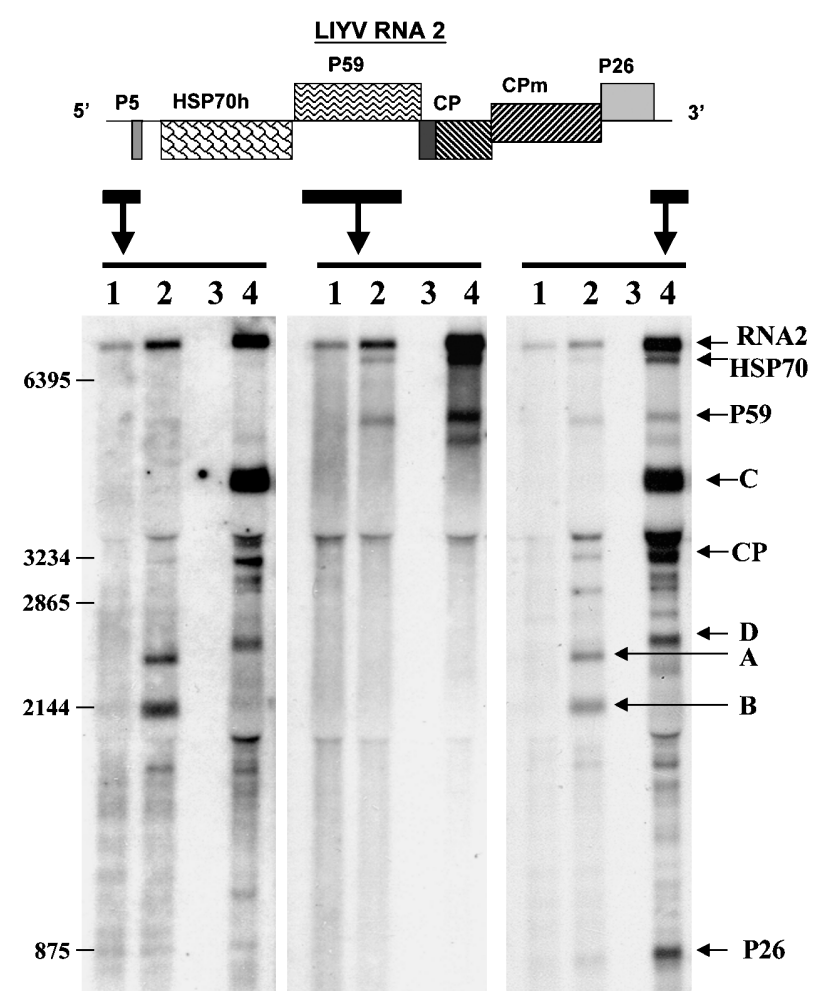

Fig. 2 Northern blot hybridization analysis of total RNAs from Nicotiana tabacum protoplasts inoculated with Lettuce infectious yellows virus (LIYV) genomic RNA 1 and RNA 2 transcripts (lanes 1 and 2, respectively), mock inoculated (lanes 3) and inoculated with plant LIYV virion RNAs (lanes 4). Total RNAs were collected $24 \mathrm{~h}$ (lanes 1) and $48 \mathrm{~h}$ (lanes 2, 3, and 4) postinoculation. A genomic map of the LIYV RNA 2 is shown at the top (Klaassen et al., 1995). Hybridizations were performed using three DIG-labelled RNA probes corresponding to different regions of the LIYV genomic RNAs (5' terminus, ORF P59, and $3^{\prime}$ terminus) indicated by lines below the LIYV genomic map. Positions of marker RNAs are given on the left. The LIYV genomic RNA 2 and specific LIYV subgenomic RNAs (HSP70h, P59, CP, P26) and defective RNAs (D RNAs) ( $A, B, C, D)$ are indicated by arrows. The shadows and resulting bands below D RNA B and above the CP subgenomic RNA are not D RNAs, but result from ribosomal RNA trapping.

to either the LIYV genomic or subgenomic RNAs (Fig. 1, inoculations 1-3, lanes 48). Interestingly, the hybridization intensities and sizes of these RNAs were different for each LIYV protoplast RNA replicate (Fig. 1, inoculations 1-3, lanes 48). Therefore, we investigated whether these RNAs could be LIYV D RNAs similar to those previously found in LIYV-infected plants (Rubio et al., 2000).

LIYV genomic RNA 1 and RNA 2 transcripts, as well as LIYV virion RNAs (virions were purified from LIYV-infected Nicotiana clevelandii plants and contained natural D RNAs), were inoculated to protoplasts. To identify LIYV D RNAs from these protoplasts, we performed Northern blot analysis on total RNAs extracted at 24 and $48 \mathrm{~h}$ p.i. using three probes corresponding to the LIYV RNA 2 gene encoding p59 and the RNA $25^{\prime}$ and $3^{\prime}$ terminal sequences (Fig. 2). As expected, the amount of LIYV genomic 
RNA 2 increased between 24 and $48 \mathrm{~h}$ p.i., indicating replication for the transcript inocula (Fig. 2, lanes 1 and 2). At $48 \mathrm{~h} \mathrm{p.i.,} \mathrm{RNAs}$ corresponding to the subgenomic RNAs for HSP70h, p59, CP, and P26 open reading frames (ORFs) were detected (Fig. 2, lanes 2 and 4). These were identified by comparing hybridization patterns for the three probes used here, and see Rubio et al. (2000). In addition to genomic and subgenomic RNAs, we also detected RNAs that hybridized with both 5' and 3' LIYV RNA 2 termini probes, but not with the p59 gene probe, indicating a typical LIYV D RNA structure (Rubio et al., 2000). The LIYV virion RNAinoculated protoplasts showed several D RNAs (e.g. C and D in Fig. 2) that corresponded in size with D RNAs reported previously for LIYV-infected plants (Rubio et al., 2000). The LIYV transcript-inoculated protoplasts showed D RNAs (e.g. A and B in Fig. 2) different from those found in LIYV virion RNA-inoculated protoplasts. Mock-inoculated protoplasts showed no hybridization signals (Fig. 2, lane 3 ).

\section{Different LIYV-inoculated protoplast replicates generate different D RNA populations}

We next assessed whether the pattern of D RNA accumulation was consistent for different LIYV protoplast RNA replicates. To do this, one LIYV RNA 1 transcript preparation (T1) and two separate RNA 2 transcript preparations (T2a and T2b) were prepared. Three protoplasts replicates $(A, B$ and $C)$ were inoculated with equivalent amounts of $T 1$ and $T 2 a$ and four $(D, E, F$ and $G$ ) with $\mathrm{T} 1$ and T2b. At $72 \mathrm{~h}$ p.i., RNAs were extracted from these seven LIYV protoplast RNA replicates $(A-G)$ and used for reverse transcriptase polymerase chain reaction (RT-PCR). We used RT-PCR for these analyses because it is more sensitive than
Northern hybridization analysis and yields products which can then be used for sequence analysis (Rubio et al., 2000). Reverse transcription was performed on all templates using the primer Rmm501, corresponding to LIYV RNA $23^{\prime}$ terminus (Table 1). PCR was then performed using primers Rmm501 and Rmm502T3, corresponding to LIYV RNA $25^{\prime}$ terminus (Table 1), but also containing a T3 RNA polymerase sequence. Because, under our PCR conditions, it was not possible to amplify the LIYV RNA 2 fulllength (size, 7193 nucleotides (nt); and see Fig. 3, lane 2), we also PCR amplified the CDNAs using the internal primers, $C P f$ and $C P r$ (Table 1). In all samples, when the CPf and CPr primers were used, a cDNA of $588 \mathrm{nt}$, the expected size, was obtained (e.g. see Fig. 3, lane 3), indicating that the RNAs were good templates for RT-PCR.

To avoid the detection of false D RNAs that might result from RT-PCR, we used as a control a mixture of LIYV RNA 1 and RNA 2 transcripts $(\mathrm{T} 1+\mathrm{T} 2 \mathrm{a})$ and total RNAs extracted $0 \mathrm{~h}$ p.i. from protoplast RNA replicates $A, B$, and C. No CDNA species were observed when RT-PCR was performed on these templates with primers Rmm501 and Rmm502T3 (Fig. 3, lanes 2, 4, 6 and 8), indicating that RT-PCR artefacts were not generated by our approach. However, from total RNAs collected $72 \mathrm{~h}$ p.i. from protoplast RNA replicates $A, B, C, D, E, F$ and $G$, one or more CDNA species ranging in size between 1 and $3 \mathrm{~kb}$ were seen for each protoplast RNA replicate (Fig. 3, lanes 5, 7, 9, 10, 11, 12 and 13). Curiously, each protoplast RNA replicate showed a distinct D RNA pattern or population. For example, protoplast RNA replicate A showed only one detectable product (Fig. 3, lane 5), whereas the remaining protoplast RNA replicates yielded products of more than one size (Fig. 3, lanes 7, 9, 10,11, 12 and 13). If the intensity of each RT-PCR electrophoretic band reflects the original

Table 1 Oligonucleotide primers used for the analysis of Lettuce infectious yellows virus (LIYV) defective RNAs (D RNAs)

\begin{tabular}{|c|c|c|}
\hline Primer & Position* & Sequence \\
\hline Rmm501† & $3^{\prime}$ terminus & 5'-ATAAGAATGCGGCCGCGGTCTAGTATACGAGATACA-3' \\
\hline Rmm502T3† & $5^{\prime}$ terminus & 5'-TGCAGAGCTCAATTAACCCTCACTAAAGGTAATCACAATTACCATTG-3' \\
\hline CPf & $4270^{*}$ & 5'-GGAATAATGATGATAAGAAGAAAGA-3' \\
\hline $\mathrm{CPr}$ & $4850^{*}$ & 5'-CATCAGTGGGTGTCGTTACCAAAACC-3' \\
\hline 2L521f & $521^{*}$ & 5'-ATGTGCAGGTATATTGTAAA-3' \\
\hline 2L1077r & $1095^{*}$ & 5'-ATGAGCTGGCTAACAACCT-3' \\
\hline 2L4158f & $4158^{*}$ & 5'-AACATTGACATTTACCTAGATTTCC-3' \\
\hline 2L963f & $963^{*}$ & 5'-AAATACGTAGCAGAGTTGGTTGAAG-3' \\
\hline $2 \mathrm{~L} 5834 \mathrm{r}$ & $5853^{*}$ & 5'-ATTAGACTCAACATCAATTG-3' \\
\hline $2 \mathrm{~L} 6234 \mathrm{r}$ & $6253^{*}$ & 5'-CGTCGTCAA AGCTGTGGTCT-3' \\
\hline 2L6751r & $6770^{*}$ & 5'-ATTTTTATTTTTAGAATATT-3' \\
\hline
\end{tabular}

*Position of first nucleotide in primers corresponding to the nucleotide sequence of LIYV RNA 2 (Klaassen et al., 1995).

tPrimer Rmm501 contains a NotI site (bold nucleotides) complementary to the 20 3'-terminal nucleotides of LIYV RNA 2 (nucleotides underlined). Primer Rmm502T3 includes an Sstl site (in bold), T3 promoter sequence (nucleotides in italics), and the 20 5'-terminal nucleotides of LIYV RNA 2 (nucleotides underlined; Klaassen et al., 1996).

$\ddagger^{\prime} f$ ' and ' $r$ ' indicate primer orientation. ' $f$ ' indicates that the primer is of the same polarity as LIYV RNA $2 .{ }^{\prime} r$ ' indicates that the primer is complementary to LIYV RNA 2. 


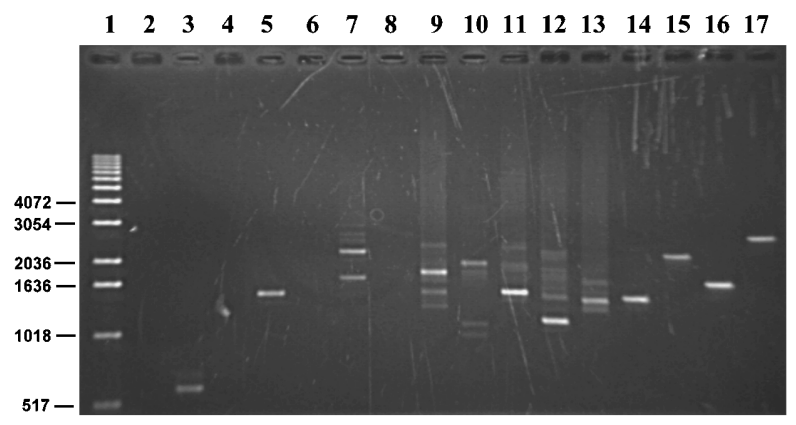

Fig. 3 Ethidium bromide-stained 1.5\% agarose gel showing reverse transcriptase polymerase chain reaction (RT-PCR) and PCR products from Lettuce infectious yellows virus (LIYV) RNA 2 defective RNAs (D RNAs) generated de novo in Nicotiana tabacum protoplasts, and cloned cDNAs. For the RT reaction, we used the primer Rmm501 (Table 1). Lane 3 corresponds to $P C R$ reactions performed with CPf and CPr (Table 1) and the remaining lanes correspond to PCR with primers Rmm501 and Rmm502T3 (Table 1). The templates used were LIYV RNA 2 transcript preparation T2a (lanes 2 and 3 ) and total RNAs extracted $0 \mathrm{~h}$ (lanes 4, 6 and 8 ) or $72 \mathrm{~h}$ (lanes 5, 7, 9, 10, 11, 12 and 13) after inoculation of $N$. tabacum protoplast preparations (A, B, C, D, E, $F$ and $\mathrm{G}$ ) with transcript preparations $\mathrm{T} 1$ and $\mathrm{T} 2 \mathrm{a}$, or $\mathrm{T} 1$ and $\mathrm{T} 2 \mathrm{~b}$, respectively. $A$ (lanes 4 and 5), B (lanes 6 and 7), and C (lanes 8 and 9) were inoculated with $T 1$ plus $T 2 a$, whereas $D, E$, F, and $G$ (lanes 10, 11, 12 and 13, respectively) were inoculated with T1 plus T2b. The CDNA clones obtained from the RT-PCR products shown in lanes 5 and 7 were PCR amplified using the primers Rmm501 and Rmm502T3. These clones were: A1 (lane 14) from protoplast $A$ RT-PCR products (see lane 5), and clones B1 (lane 15), B6 (lane 16), and B14 (lane 17) from protoplast B RT-PCR products (see lane 7). The DNA size marker, $1 \mathrm{~kb}$ DNA ladder (GibcoBRL), is shown in lane 1 and the sizes of the corresponding DNAs are indicated on the left.

proportion of the corresponding template, and if the products represent LIYV RNA 2 D RNAs, then the patterns suggest that, within each protoplast RNA replicate, D RNAs are generated and/ or accumulate differently. But, within each protoplast RNA replicate, one or only a few D RNAs appeared to be predominant.

\section{Characterization of LIYV RNA 2 D RNAs generated de novo}

The RT-PCR products obtained from total RNAs extracted at $72 \mathrm{~h}$ p.i. from protoplast RNA replicates A and B (see Fig. 3, lanes 5 and 7) were cloned. Seven clones from isolate $A$ and 14 clones from isolate $B$ were used as templates for PCR using primers Rmm501 and Rmm502T3 (Table 1), and all yielded a single DNA product. The seven clones from protoplast RNA replicate $A$ all contained a CDNA insert indistinguishable in size from that of the original RT-PCR product (see Fig. 3, lanes 5 and 14). One clone (designated A1) was saved for further analysis (Fig. 3, lane 14). For protoplast RNA replicate $B$, three different sized DNAs were obtained (named B1, B6, and B14). Seven clones gave B1, six gave B6, and one gave B14 (Fig. 3, lanes 15, 16 and 17, respect-

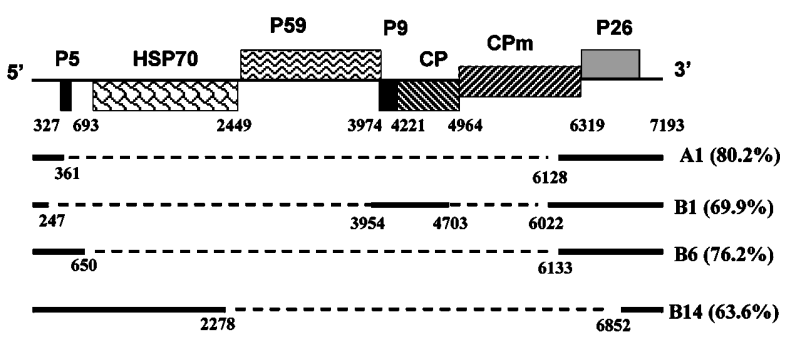

Fig. 4 Schematic representation of cloned CDNA obtained from Lettuce infectious yellows virus (LIYV) defective RNAs (D RNAs) generated de novo in Nicotiana tabacum protoplasts (electrophoretic patterns of PCR products of these D RNAs are shown in lanes 14-17 in Fig. 3). The LIYV genomic RNA 2 map is indicated at the top. Numbers below the map indicate the nucleotide position of the corresponding open reading frame (ORF) start codons. 7193 is the position of the LIYV RNA $23^{\prime}$-most nucleotide. Continuous lines represent nucleotide sequences of D RNAs common to LIYV RNA 2. Discontinuous lines correspond to deleted regions not present in the corresponding D RNAs. Numbers given below the lines indicate the LIYV RNA 2 D RNA junction positions. The deletion sizes (percentage with respect to the full-length genomic RNA) are indicated in parentheses.

ively). The sizes of B1, B6, and B14 corresponded to those of the three major DNA species seen for the original RT-PCR products (Fig. 3, lane 7). The number of clones for each CDNA species was in agreement with the relative intensity of the corresponding original RT-PCR product (Fig. 3, lane 7).

To determine whether these DNAs represented D RNAs, the nucleotide sequences of two clones for each DNA species (A1, $\mathrm{B} 1 \mathrm{~B} 6$, and B14) were determined. In all cases, clones corresponding to the same CDNA species showed identical nucleotide sequences. When the nucleotide sequences of these clones were compared with that of LIYV genomic RNA 2 (Klaassen et al., 1995), they showed a typical D RNA structure (Rubio et al., 2000). Thus, these clones had corresponding $5^{\prime}$ and $3^{\prime}$ termini of the LIYV RNA 2, but showed extensive internal nucleotide sequence deletions (Fig. 4). The nucleotide identities between the overlapping sequences of LIYV genomic RNA 2 and these four D RNAs (A1, B1, B6 and B14) were greater than $99 \%$. The sizes of the LIYV RNA 2 terminal sequences varied for different CDNA clones, ranging from 247 to $2278 \mathrm{nt}$ for the $5^{\prime}$ terminus, and from 341 to $1171 \mathrm{nt}$ for the $3^{\prime}$ terminus, and the four D RNAs contained one or two intact ORFs. D RNA A1 had a central deletion corresponding to $c .80 \%$ of LIYV RNA 2 . In the junction site, there was a sequence with the nucleotides TTAGAATG, repeated in the genomic RNA 2 positions 316 and 6128, where the deletion occurred. Both repeats were not identical, as the nucleotide stretch in position 316 had a $T$ instead of $A$ at the fifth nucleotide. The other D RNAs had deletions similar in size to that of $A 1$, but no repeats were identified in the genomic RNA. Interestingly, the D RNA B1 showed two deletions whose junction sites were in positions 247/3954 and 4703/6022 (Fig. 4). 


\section{DISCUSSION}

We synthesized in vitro transcripts from full-length genomic LIYV RNA 1 and RNA 2 CDNA clones and inoculated them to different protoplast replicates. The accumulation of LIYV genomic and subgenomic RNAs was accompanied by the rapid appearance of LIYV RNA 2-derived D RNAs. Because the inoculum contained only LIYV genomic RNAs 1 and 2, the D RNAs detected here must have been generated de novo. Some of these D RNAs accumulated so rapidly that, at $48 \mathrm{~h}$ p.i., they were detected by Northern blot analysis. This contrasts with other plant viruses that showed detectable D RNAs only after serial passages (Graves and Roossinck, 1995; Knorr et al., 1991), or after prolonged infections (Damayanti et al., 1999; Law and Morris, 1994).

The D RNAs from LIYV-inoculated protoplast replicates (generated de novo) also showed some differences with respect to the D RNAs previously analysed from LIYV-infected plants (Rubio et al., 2000). The LIYV-infected plant D RNA population was much more diverse (greater number of different D RNAs) than the protoplast D RNA populations analysed here. It is very likely that the plant D RNAs have undergone many replication cycles, as our LIYV isolate has been maintained for many years by serial passages in plants by its whitefly vector. Many replication cycles within individual and different plants theoretically would allow numerous opportunities for D RNAs to arise. These D RNAs, however, would still be under selection for replication competence, but probably also for encapsidation, the ability to move within the plant host, and the ability to be transmitted from plant to plant by $B$. tabaci. Of the 33 different plant D RNAs previously characterized by us (Rubio et al., 2000), all had conserved LIYV RNA $25^{\prime}$ and $3^{\prime}$ termini (but of different lengths) and one large central deletion (but varying in size) that always included the HSP70h and p59 ORFs (Rubio et al., 2000). Although the individual protoplast RNA replicates showed less D RNA diversity, two new D RNA types not detected in the plant-derived D RNAs were found. Protoplast $D$ RNA B14 conserved most of the HSP70h ORF (see Fig. 4), and protoplast D RNA B1 was a mosaic, corresponding to LIYV RNA 2 , but having two deleted non-contiguous genomic regions (see Fig. 4).

Several interesting questions can be raised about de novo generated D RNAs, including: how are they formed?; how do they accumulate?; and why did each protoplast RNA replicate generate a different $D$ RNA population? The mechanisms for the generation of plant virus D RNAs have not been definitively demonstrated. The 'copy choice' mechanism is the most accepted model. In this model, the D RNAs are generated by jumping of the replicase-nascent strand complex from one position on the template to another position on the same or another RNA molecule (Nagy and Simon, 1997). It is believed that the dissociation and re-association of the replicase-nascent strand complex does not occur randomly. Dissociation may be induced by strong secondary structure or weak base pairing within the A/U-rich regions. Re-association can be aided by motifs in the receptor template, such as short nucleotide stretches complementary with the incomplete nascent strand, a bubble formed by weak pairing in A/U-rich sequences, secondary structure, etc. (Nagy and Bujarski, 1997; White and Morris, 1999). However, D RNA replication competence rather than D RNA generation is believed to be the main factor in D RNA accumulation. Factors such as D RNA length, conservation of a complete ORF, presence or absence of internal cis-acting motifs, secondary structure, etc. have also been reported to influence D RNA replication competence (Knapp et al., 2001; Mawassi et al., 2000; White and Morris, 1999; Yeh et al., 2001). Thus, differential generation and/or accumulation of D RNAs could account for the D RNA pattern (D RNAs of different concentration) observed in each LIYV protoplast RNA replicate. It is also very intriguing that separate replicates of the same protoplast preparation inoculated with separate aliquots of the same LIYV RNA 1 and RNA 2 transcripts yielded different D RNA populations. One possible explanation is that de novo D RNA generation and/or accumulation processes might be very sensitive to minute variations in the initial conditions, typical of non-linear systems where the different factors affect one another in a synergistic manner (Lorenz, 1996).

Another interesting question concerns the biological significance of the D RNAs in the virus infection. Whether or not D RNAs are useful for the virus or are simply erroneous products of genetic recombination (Roossinck, 1997; Simon and Bujarski, 1994) is not known. There does not appear to be a strong negative selection against LIYV D RNA formation and accumulation as no noticeable interference with LIYV genomic RNAs has been observed (Rubio et al., 2000). In contrast, recombination could confer advantages and could act as a compensatory mechanism to offset the accumulation of deleterious mutations and allow greater genome diversity and adaptability to new environments (Roossinck, 1997). Recombination might be especially important for viruses of the family Closteroviridae, as they have the largest genomes among the known single-stranded plus-sense RNA plant viruses and, hence, may be at greater risk for the accumulation of deleterious mutations. This could partly explain the great number and rapid appearance of D RNAs observed with LIYV (Rubio et al., 2000) and for Citrus tristeza virus (CTV, genus Closterovirus of the family Closteroviridae) (Ayllón et al., 1999; Mawassi et al., 1995).

\section{EXPERIMENTAL PROCEDURES}

\section{In vitro transcription and protoplast inoculation}

Full-length RNA transcripts were in vitro synthesized from CDNA clones of LIYV genomic RNA 1 and RNA 2 (Klaassen et al., 1996) with T3 RNA polymerase using mMESSAGE mMACHINE Kit 
(Ambion) according to the manufacturer's instructions. LIYV RNA 1 and RNA 2 transcripts and LIYV virion RNAs were inoculated to protoplasts prepared from Nicotiana tabacum suspension cells and incubated as previously described (Yeh et al., 2000).

\section{RNA analysis}

LIYV virions and LIYV virion RNAs were purified from LIYVinfected $N$. clevelandii plants and analysed as previously described (Klaassen et al., 1994). Total RNAs from LIYV virion RNA- and LIYV RNA 1 and RNA 2 transcript-inoculated N. tabacum protoplasts were isolated using TRI Reagent (MRO) according to the manufacturer's instructions. The RNAs were denatured and analysed by Northern hybridization using DIG-labelled RNA probes corresponding to specific genomic regions of LIYV RNA 2 (see Fig. 2) as previously described (Klaassen et al., 1994, 1995, 1996).

\section{Reverse transcription and polymerase chain reaction, cloning, and nucleotide sequence analysis}

LIYV in vitro transcripts and protoplast total RNAs were used as templates for the synthesis of CDNAs (reverse transcription) using the oligonucleotide Rmm501 (Table 1) and 40 U Superscript II Rnase $\mathrm{H}$ - (Gibco) according to the manufacturer's instructions. The CDNAs were PCR amplified in a $20 \mu \mathrm{L}$ reaction mixture containing PCR buffer, $2 \mathrm{~mm} \mathrm{MgSO}_{4}, 1 \mathrm{~mm}$ of each of the four dNTPs (deoxynucleotide triphosphates), $0.4 \mathrm{U}$ Platinum Taq High Fidelity (Gibco), and $50 \mathrm{ng}$ of each oligonucleotide: Rmm501 and Rmm502T3 (Table 1). After an initial denaturing step at $94^{\circ} \mathrm{C}$ for $4 \mathrm{~min}$, PCR was performed for 30 cycles, each of $94^{\circ} \mathrm{C}$ for $30 \mathrm{~s}$, $50^{\circ} \mathrm{C}$ for $30 \mathrm{~s}$, and $72{ }^{\circ} \mathrm{C}$ for $8 \mathrm{~min}$, followed by an extension step at $72{ }^{\circ} \mathrm{C}$ for $10 \mathrm{~min}$. RT-PCR products were separated by electrophoresis on $1.5 \%$ agarose gels and detected by ethidium bromide staining. RT-PCR products were cloned into PGEM-T (Promega) by incubating with DNA ligase (Promega) for $1 \mathrm{~h}$ at room temperature, followed by transformation into Escherichia coli, $\mathrm{DH} 5 \alpha$ (Sambrook et al., 1989). Nucleotide sequences of the cloned CDNAs were determined in both directions using primers corresponding to the T7 and SP6 plasmid promoters and the ABI PRISM DNA sequencer 377 (Perkin-Elmer, Foster City, CA, USA). Additionally, a set of specific LIYV RNA 2 primers was used to determine internal nucleotide sequences (primers 2L521f, 2L4158f, 2L963f, 2L5834r, 2L6234r and 2L6751r in Table 1). Nucleotide sequences were aligned with the program GAP from the Wisconsin GCG software package (Devereux et al., 1984).

\section{ACKN OWLEDGEMENTS}

This research was supported in part by grants from the U.S.D.A. NRICGP and the University of California.

\section{REFERENCES}

Ayllón, M.A., López, C., Navas-Castillo, J., Mawassi, M., Dawson, W.O., Guerri, J., Flores, R. and Moreno, P. (1999) New defective RNAs from citrus tristeza virus: evidence for a replicase-driven template switching mechanism in their generation. J. Gen. Virol. 80, 817-821.

Damayanti, T.A., Nagano, H., Mise, K., Furusawa, I. and Okuno, T. (1999) Brome mosaic virus defective RNAs generated during infection of barley plants. J. Gen. Virol. 80, 2511-2518.

Devereux, J., Haeberli, P. and Smithies O. (1984) A comprehensive set of sequence analysis programs for the VAX. Nucl. Acids Res. 12, 387395.

Graves, M.V., Pogany, J. and Romero, J. (1996) Defective interfering RNAs and defective viruses associated with multipartite RNA viruses of plants. Semin. Virol. 7, 399-408.

Graves, M.V. and Roossinck, M.J. (1995) Characterization of defective RNAs derived from RNA 3 of the Fny strain of cucumber mosaic cucumovirus. J. Virol. 69, 4746-4751.

Holland, J.J. (1990) Defective viral genomes. In: Virology (Fields, B.N. and Knipe, D.M., eds). New York: Raven Press, pp. 151-165.

Klaassen, V.A., Boeshore, M., Dolja, V.V. and Falk, B.W. (1994) Partial characterization of the lettuce infectious yellows virus genomic RNAs, identification of the coat protein gene and comparison of its amino acid sequence with those of other filamentous RNA plant viruses. J. Gen. Virol. 75, 1525-1533.

Klaassen, V.A., Boeshore, M.L., Koonin, E.V., Tian, T. and Falk, B.W. (1995) Genome structure and phylogenetic analysis of lettuce infectious yellows virus, a whitefly-transmitted, bipartite closterovirus. Virology, 208, 99-110.

Klaassen, V.A., Mayhew, D., Fisher, D. and Falk, B.W. (1996) In vitro transcripts from cloned CDNAs of the lettuce infectious yellows closterovirus bipartite genomic RNAs are competent for replication in Nicotiana benthamiana protoplasts. Virology, 222, 169-175.

Knapp, E., Dawson, W.O. and Lewandowski, D.J. (2001) Conundrum of the lack of defective RNAs (dRNAs) associated with tobamovirus infections: dRNAs that can move are not replicated by the wild-type virus; dRNAs that are replicated by the wild-type virus do not move. J. Virol. 75, $5518-5525$.

Knorr, D.A., Mullin, R., Hearne, P.Q. and Morris, T.J. (1991) De novo generation of defective interfering RNAs of tomato bushy stunt virus by high multiple passage. Virology, 181, 193-202.

Law, M.D. and Morris, T.J. (1994) De novo generation and accumulation of tomato bushy stunt virus defective interfering RNAs without serial host passage. Virology, 198, 377-380.

Lorenz, E.N. (1996) The Essence of Chaos. Seattle, WA: University of Washington Press.

Mawassi, M.E., Mietkiewska, M.E., Hilf, L., Ashoulin, A., Karasev, V., Gafny, R., Lee, R.F., Garnsey, S.M., Dawson, W.O. and Bar-Joseph, M. (1995) Multiple species of defective RNAs in plants infected with citrus tristeza virus. Virology, 214, 264-268.

Mawassi, M., Satyanarayana, T., Abiach-Martí, M.R., Gowda, S., Ayllón, M.A., Robertson, C. and Dawson, 0. (2000) The fitness of Citrus tristeza virus defective RNAs is affected by the lengths of their $5^{\prime}$ - and $3^{\prime}$-termini and by the coding capacity. Virology, 275, 4256.

Nagy, P.D. and Bujarski, J.J. (1997) Engineering of homologous recombination hotspots with AU-rich sequences in brome mosaic virus. J. Virol. 71, 3799-3810. 
Nagy, P.D. and Simon, A.E. (1997) New insights into the mechanisms of RNA recombination. Virology, 235, 1-9.

Roossinck, M.J. (1997) Mechanisms of plant virus evolution. Annu. Rev. Phytopathol. 35, 1953-1965.

Rubio, L., Yeh, H.-H., Tian, T. and Falk, B.W. (2000) A heterogeneous population of defective RNAs is associated with Lettuce infectious yellows virus (LIYV). Virology, 271, 205-212.

Sambrook, J., Fritsch, E.F. and Maniatis, T. (1989) Molecular Cloning. A Laboratory Manual. Cold Spring Harbor, NY: Cold Spring Harbor Laboratory.

Simon, A.E. and Bujarski, J.J. (1994) RNA-RNA recombination and evolution in virus-infected plants. Annu. Rev. Phytopathol. 32, 337-376.

Torrance, L., Cowan, G., Sokmen, M.A. and Reavy, B. (1999) A naturally occurring deleted form of RNA 2 of Potato mop-top virus. J. Gen. Virol. 80, 2211-2215.

White, K.A. and Morris, T.J. (1999) Defective and defective interfering RNAs of monopartite plus-strand RNA plant viruses. In: Satellites and Defective Viral RNAs (Vogt, P.K. and Jackson, A.O., eds). Berlin, Heidelberg: Springer-Verlag.

Yeh, H., Tian, T., Medina, V. and Falk, B.W. (2001) Green fluorescent protein expression from recombinant Lettuce infectious yellows virus RNA 2 defective RNAs. Virology, 289, 54-62.

Yeh H., Tian, T., Rubio, L., Crawford, B. and Falk, B.W. (2000) Asynchronous accumulation of Lettuce infectious yellows virus RNAs 1 and 2 and identification of an RNA 1 trans enhancer of RNA 2 accumulation. J. Virol. 74, 5762-5768. 